\title{
Toward clinical transparency
}

\author{
Big pharma has historically made some substantial missteps regarding the full reporting of clinical trial results, but a \\ new initiative by GlaxoSmithKline is a move in the right direction.
}

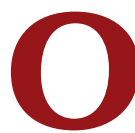
n 11 October, GlaxoSmithKline (GSK) announced that it will make detailed anonymized patient-level data from their clinical trials available to qualified researchers (http://www.gsk.com/media/press-releases/2012/ GSK-announces-further-initiatives-to-tackle-global-healthchallenges.html). According to the GSK press release announcing the initiative, the move will help further the aim of GSK to increase the transparency of its studies, and it may allow researchers "to examine the data more closely or to combine data from different studies in order to conduct further research, to learn more about how medicines work in different patient populations and to help optimise the use of medicines with the aim of improving patient care."

This announcement follows on the heels of GSK's development of a public website in 2004 that lists summary results and protocols of its trials. In view of the pharmaceutical industry's checkered history in reporting clinical trial data, GSK's further commitment to increasing openness and transparency is a welcome development. Last year, GSK was itself the subject of a US government lawsuit accusing the company of promoting Paxil and Wellbutrin for unapproved uses in children and adolescents, in spite of GSK trials-in the case of Paxil-that did not show effectiveness and raised safety concerns. In the same lawsuit, GSK was accused of promoting Advair as a superior medication for asthma, basing its assertion on a study whose claims the US Food and Drug Administration had evaluated and rejected. As a result of this lawsuit, the company agreed to pay a $\$ 3$ billion fine, although GSK told Nature Medicine that the plans for the new transparency initiative have been in the works for the past 18 months and were not related to the lawsuit.

To implement the new program, GSK will create a secure website containing detailed patient data and the full protocols of clinical trials GSK has conducted for approved and discontinued drugs. GSK plans to make the data accessible after regulatory approval and publication of the trial results in the scientific literature. For data that are associated with trials that did not lead to drug approval or that are not published, the plan is to offer access within a reasonable timeframe. The new policy will apply to global clinical trials from 2007 and to all clinical trials starting in 2013.
External access to the detailed data should provide unprecedented insight into GSK's clinical trial results, particularly for failed trials, which might otherwise not see the light of day. But access will not be open to all. According to James Shannon, the company's chief medical officer, to ensure "this information is not misused, which could be detrimental to medical science and patient care, research requests will need to be submitted with proposals, which will be reviewed for scientific merit by an independent panel of experts." But it's unknown who will decide the makeup of the panel and what criteria will determine the "scientific merit" of the proposals. Would researchers from competing companies working on similar drugs be allowed access to the detailed patient data? Moreover, a lot of money could be at stake, so the mechanisms that will be used to protect against conflicts of interest (for both the review board members and those applying to access the data) must be made transparent, and the reviews should be done in a timely manner.

With regard to the firewall, the company has expressed concern about patient privacy and that the data might be misinterpreted by nonexperts. Accordingly, the company will place certain conditions on researchers who are granted access. Namely, they must use the data only as outlined in their proposal, keep it confidential, not attempt to identify patients in the study, and inform regulatory authorities and GSK of any safety concerns they might uncover. It remains unclear how GSK would react if important safety concerns were identified and the economic interests of the company put at risk.

We applaud GSK for taking this step and hope the rest of the industry follows suit. Databases of this sort are bound to improve trial data analysis, as highly qualified outside experts will have a chance to offer their own independent assessments. They may also enable repurposing of drugs that were safe but failed to show efficacy for a particular indication, and perhaps improve future trial designs. And their utility will increase as pharmacogenomic data become more readily available. The ability of outside researchers to analyze clinical trial data should ultimately help companies learn how to better target their drugs to the patients that can benefit from them the most. 